\title{
Questes
}

\section{Éléments de bibliographie}

Sarah Delale et Jean-Dominique Delle Luche

\section{(2) OpenEdition \\ Journals}

\section{Édition électronique}

URL : http://journals.openedition.org/questes/4287

DOI : 10.4000/questes.4287

ISSN : 2109-9472

\section{Éditeur}

Les Amis de Questes

\section{Édition imprimée}

Date de publication : 31 octobre 2015

Pagination : 135-144

ISSN : 2102-7188

\section{Référence électronique}

Sarah Delale et Jean-Dominique Delle Luche, «Éléments de bibliographie », Questes [En ligne],

31 | 2015, mis en ligne le 24 janvier 2016, consulté le 30 avril 2019. URL : http://

journals.openedition.org/questes/4287; DOI : 10.4000/questes.4287 


\title{
Éléments de bibliographie
}

\author{
Sarah DeLALE, Jean-Dominique DELLE LUCHE \\ Université Paris-Sorbonne, École des Hautes Études en Sciences Sociale
}

Cette bibliographie n'a pas vocation à l'exhaustivité et reprend essentiellement les références utilisées dans le numéro. En conséquence, nous avons cru bon de classer certaines références dans plusieurs catégories différentes, afin de rendre plus intéressant l'usage de la bibliographie.

\section{Fête, jeu et calendrier : ouvrages et articles généraux}

Cabantous, Alain, Le Dimanche, une histoire. Europe occidentale (1600-1830), Paris, Seuil, coll. «L'univers historique », 2013.

Les Calendriers ; leurs enjeux dans l'espace et dans le temps. Colloque de Cerisy, $1^{\text {er }}$ 8 juillet 2000, dir. Jacques Le Goff, Jean Lefort et Perrine MANE, Paris, Somogy éditions d'art, 2002.

City and spectacle in Medieval Europe, dir. Barbara HANAWALT et Kathryn REYERSON, Minneapolis, University of Minnesota Press, coll. «Medieval studies in Minnesota », nº 5, 1994.

Cox, Harvey, La Fête des fous, essai théologique sur les notions de fête et de fantaisie, trad. Luce GIARD, Paris, Seuil, 1971.

Écrire, traduire et représenter la fête. VIII Coloquio de la Asociación de profesores de filología francesa de la universidad española, dir. Elena REAL, Dolores JIMENEZ et Domingo PujAnTE, València, Servei de Publicacions de la Universitat de València, 2001.

Feste und Feiern im Mittelalter. Paderborner Symposion des Mediävistenverbandes, dir. Detlef Altenburg, Jörg JARnut et Hans-Hugo SteInHofF, Sigmaringen, Jan Thorbecke Verlag, 1991.

Freizeit und Vergnügen vom 14. bis zum 20. Jahrhundert $=$ Temps libre et loisirs $d u$ $X I V^{e}$ au $X X^{e}$ siècle, dir. Hans-Jörg GILOMEN, Beatrice SCHUMACHER et Laurent TISSOT, Zürich, Chronos, coll. «Schweizerische Gesellschaft für Wirtschafts- und Sozialgeschichte $», n^{\circ} 20,2005$.

GlaUSER-MateCKI, Antoinette, Un Aspect du cycle de mai en Europe occidentale : rites et coutumes des calendes de mai, thèse de l'EHESS, 1996.

HeERs, Jacques, Fêtes, jeux et joutes dans les sociétés d'Occident à la fin du Moyen Âge, Paris, Vrin, 1971. 
JaCQuOT, Jean, Les Fêtes de la Renaissance. 2. Fêtes et cérémonies au temps de Charles Quint, Paris, Éditions du CNRS, coll. « Le chœur des muses », 1973.

Jeux, sports et divertissements au Moyen Âge et à l'Âge classique, Paris, Éditions du CTHS (Actes du 116e Congrès des Sociétés savantes, Chambéry 1991), 1993.

LECUPPRE-DESJARDIN, Élodie, La Ville des cérémonies. Essai sur la communication politique dans les anciens Pays-Bas bourguignons, Turnhout, Brepols, coll. «Studies in European urban history », vol. 4, 2004.

MAfFESOl, Michel, «Communion et communication. Penser le mystère de la sociabilité contemporaine », Sociétés, 2006/1, nº 91, p. 7-10.

MANE, Perrine, La Vie dans les campagnes au Moyen Âge à travers les calendriers, Paris, la Martinière, 2004.

NADOT, Sébastien, Le Spectacle des joutes. Sport et courtoisie à la fin du Moyen Âge, Rennes, Presses Universitaires de Rennes, coll. « Histoire », 2012.

WALTER, Philippe, La Mémoire du temps, fêtes et calendriers de Chrétien de Troyes à la Mort Artu, Paris/Genève, Champion/Slatkine, coll. « Nouvelle bibliothèque du Moyen Âge », n 13, 1989.

-, Mythologie chrétienne: Fêtes, rites et mythes du Moyen Âge, Paris, Imago, coll. « Le grand livre du mois », 2003.

\section{D'une fête à l'autre}

\section{Fête et des fous, carnaval et charivari}

Bertrand, Gilles, Histoire du carnaval de Venise : XI $I^{e} X X I^{e}$ siècles, Paris, Pygmalion, 2013.

BURKE, Peter, «Le carnaval de Venise : esquisse pour une histoire de longue durée », dans Les Jeux à la Renaissance, dir. Philippe ARIES, Paris, Vrin, 1982, p. 55-61.

Le Charivari, dir. Jacques LE GoFf et Jean-Claude SchmiTt, Paris/La Haye/New York, Mouton, EHESS, coll. «Civilisations et Sociétés », n 67, 1981.

HeERs, Jacques, Fêtes des fous et carnavals, Paris, Fayard, coll. «Pluriel », 1983.

LE PERSON, Marc, «Entre critique, dérision et subversion de l'autorité et de la loi : Le jeu de la feuillée d'Adam de la Halle (XIII siècle) est-il une Fête des Fous dramatisée? », Le Moyen Âge et la Renaissance face aux lois : de la critique à la subversion, Cahiers du CELEC, $\mathrm{n}^{\circ}$ 8, 2014.

MäND, Anu, Urban Carnival: festive culture in the hanseatic cities of the eastern Baltic, 1350-1550, Turnhout, Brepols, coll. «Medieval texts and cultures of Northern Europe », n 8, 2005.

PlaisAnce, Michel, «Le carnaval des Médicis: de Laurent à François » dans Florence and Milan. Comparisons et relations, dir. Craig Hugh SMYTH et Giancarlo GARFAGNINI, Firenze, La Nuova Italia editrice, coll. «Villa i Tatti », n 11,1989, vol. 2, p. 243-255.

ReY-Flaud, Henri, Le Charivari. Les rituels fondamentaux de la sexualité, Paris, Payot, 1985. 


\section{Nouvel an}

HIRSCHBIEGEL, Jan, «Zeichen der Gunst : Neujahrsgeschenke am burgundischen Hof zur Zeit König Karls VI. von Frankreich (1380-1422)», dans Menschenbilder Menschenbildner: Individuum und Gruppe im Blick des Historikers. Werner Paravicini zum 60. Geburtstag, dir. Stephan SELZER, Berlin, Akademie-Verlag, coll. «Hallische Beiträge zur Geschichte des Mittelalters und der Frühen Neuzeit », $\mathrm{n}^{\mathrm{o}} 2,2002$, p. 213-240.

\section{Tournois et joutes}

BOUSMAR, Éric, «La place des hommes et des femmes dans les fêtes de cour bourguignonnes (Philippe le Bon - Charles le Hardi)», dans $\grave{A}$ la cour de Bourgogne. Le duc, son entourage, son train, dir. Jean-Marie CAUCHIES, Turnhout, Brepols, coll. «Burgundica », $\mathrm{n}^{\circ}$ 1, 1998, p. 11-31.

HEERS, Jacques, Fêtes, jeux et joutes dans les sociétés d'Occident à la fin du Moyen Âge, Paris, Vrin, 1971.

NAdot, Sébastien, Le Spectacle des joutes. Sport et courtoisie à la fin du Moyen Âge, Rennes, Presses Universitaires de Rennes, coll. « Histoire », 2012.

RANFT, Andreas, «Die Turniere der vier Lande: Genossenschaftlicher Hof und Selbstbehauptung des niederen Adels », Zeitschrift für Geschichte des Oberrheins, $\mathrm{n}^{\mathrm{o}} 142,1994$, p. 83-102.

VAN DEN NESTE, Évelyne, Tournois, joutes, pas d'armes dans les villes de Flandre à la fin du Moyen Âge (1300-1486), Paris, École des Chartes, coll. «Mémoires et documents de l'École des Chartes », nº 47, 1996.

\section{Tirs}

CAuchiES, Jean-Marie, "Service" du prince, "sûreté" des villes : à propos de privilèges délivrés aux confréries ou serments d'archers et d'arbalétriers dans les Pays-Bas au XV siècle », Revue du nord, 2012, vol. 94, no 395, 2012, p. 419-434.

DELlE LUCHE, Jean-Dominique, «Schützenfeste und Schützengesellschaften in den Residenzstädten: Konfigurationen zwischen Stadt und Fürsten im 15. und 16. Jahrhundert (Pforzheim, Würzburg, Ansbach, Stuttgart)», dans In der Residenzstadt. Funktionen, Medien, Formen bürgerlicher und höfischer Repräsentation, dir. Jan HIRSCHBIEGEL et Werner PARAVICINI, Ostfildern, Thorbecke, 2014, p. 157-174.

EDElmann, August, Schützenwesen und Schützenfeste der deutschen Städte vom 13. bis zum 18. Jahrhundert, München, Pohl, 1890.

KuZudo, Kazuhiko, «Open Shooting Festivals (Freischießen) in German Cities, 1455-1501 », The International Journal of the History of Sport, vol. 3, n 1, 1999 p. $65-86$.

MeHL, Jean-Michel, «Une éducation du corps à la fin du Moyen Âge et au début de la Renaissance : le tir à l'arc en France et en Angleterre »dans Éducation et hygiène 
du corps à travers l'histoire. Actes du colloque de l'Association interuniversitaire de l'Est (Dijon, 26 et 27 septembre 1989), dir. Pierre LEVEQUE, Dijon, EUD, coll. «Publications de l'Association Interuniversitaire de l'Est », 1991, p. 17-27.

OSTERMAnN, Markus, " "Vmb kurczweil vnd schiessens willen". Zu den gedruckten Schützenbriefen des 15. Jahrhunderts », dans Einblattdrucke des 15. und frühen 16. Jahrhunderts : Probleme, Perspektiven, Fallstudien, dir. Volker HonEMANN, Tübingen, Niemeyer, 2000, p. 397-443.

RADLKOFER, Max, «Die Schützengesellschaften und Schützenfeste Augsburgs im 15. und 16. Jahrhundert », Zeitschrift des Historischen Vereins für Schwaben und Neuburg, n $^{\circ} 21,1894$, p. 87-139.

\section{Jeux d'eau}

Gouedo-Thomas, Catherine, «Natation et joutes nautiques à travers l'iconographie des manuscrits à peintures ( $\mathrm{XIII}^{\mathrm{e}}-\mathrm{XVI}^{\mathrm{e}} \mathrm{s}$.) $)$ dans Jeux, sports et divertissements au Moyen Âge et à l'Âge classique. Actes du $116^{\mathrm{e}}$ Congrès National des Sociétés Savantes, Chambéry, 1991, p. 156-175.

\section{Jeux d'hiver}

AleXANDRE-BIDON, Danièle, «Les jeux et sports d'hiver au Moyen Âge et à la Renaissance », dans Jeux, sports et divertissements au Moyen Âge et à l'Âge classique, Paris, Éditions du CTHS (Actes du $116^{\mathrm{e}}$ Congrès des Sociétés savantes, Chambéry 1991), 1993, p. 143-156.

\section{Les fêtes de la Croix}

BROWN, Peter, Le Culte des saints : son essor et sa fonction dans la chrétienté latine, trad. Aline Rousselle, Paris, Éditions du Cerf, coll. « Histoire », nº 2, 1996.

CORnET, Bernard, «La fête de la Croix du 3 mai », Revue belge de philologie et d'histoire, vol. 30, $\mathrm{n}^{\mathrm{o}} 3-4,1952$, p. 837-848.

JANERAS, Sebastia, Le Vendredi Saint dans la tradition liturgique byzantine : structure et histoire de ses offices, Roma, Pontificio ateneo S. Anselmo, coll. "Studia Anselmiana $» n^{\circ} 99$ et «Analecta liturgica », $n^{\circ} 12,1988$.

Jounel, Pierre, «Le culte de la Croix dans la liturgie romaine », La maison Dieu, $\mathrm{n}^{\mathrm{o}} 75,1963$, p. 68-91.

LECLERCQ, Henri, «Croix (invention et exaltation de la vraie) », dans Dictionnaire d'archéologie chrétienne et de liturgie, dir. Henri LECLERCQ et Fernand CABROL, Paris, Letouzey et Ané, 1914, vol. 3, part. 2, col. 3131-3139.

QUILLIET, Hector, «Croix (adoration de la)», dans Dictionnaire de théologie catholique, dir. Alfred VACANT, Eugène MAngEnot et Emile Amann, Paris, Letouzey et Ané, 1923, t. III, part. 2, col. 2339-2363. 
VAN TONGEREN, Louis, Exaltation of the Cross: toward the origins of the feast of the Cross and the meaning of the Cross in early Medieval liturgy, London, Peeters, coll. «Liturgia condenda », $\mathrm{n}^{0} 11,2000$.

\section{Entrées royales et tableaux vivants}

BlAnCHARD, Joël, «Le spectacle du rite: les entrées royales » Revue Historique, $n^{\circ} 627$, t. CCCV/03, 2003, p. 475-519.

BlockmAns, Wim, «Le dialogue imaginaire entre princes et sujets : les joyeuses entrées en Brabant en 1494 et en 1496 », dans À la cour de Bourgogne. Le duc, son entourage, son train, dir. Jean-Marie CAUCHIES, Turnhout, Brepols, coll. «Burgundica », $\mathrm{n}^{0}$ 1, 1998, p. 155-170.

BONICEL, Mathieu, «Les modes de financement public des performances à Avignon à la fin du Moyen Âge », Studies in Early Modern France, vol. 13, «Spectacle», 2010, p. 31-40.

BRYANT, Lawrence M., The King and the city in the parisian royal entry ceremony, Genève, Droz, coll. «Travaux d'humanisme et Renaissance », nº 216, 1986.

-, Ritual, ceremony and the changing monarchy in France, 1350-1789, Farnham, Ashgate, coll. «Collected studies series », $\mathrm{n}^{\circ}$ 937, 2010.

COLLARD, Franck, «Fête du prince ou Fête de Reims : l'entrée de Charles VIII à Reims le 29 mai 1484 » dans Fêtes et politique en Champagne à travers les siècles, dir. Sylvette GuILBERT, Nancy, Presses Universitaires de Nancy, coll. «Études champenoises », 1992, p. 59-71.

GuENEE, Bernard et Françoise LeHOuX, Les Entrées royales françaises de 1328 à 1515, Paris, Éditions du CNRS, coll. «Sources de l'histoire médiévale », n 5 , 1968.

JOUBERT, Fabienne, «Les tableaux vivants et l'Église » dans Le Théâtre de l'Église (XII $-X V I^{e}$ siècles), dir. Marie BOUHAÏK-GIRONES, Paris, LAMOP, 2011, en ligne sur https://lamop.univ-paris1.fr/IMG/pdf/FJoubert.pdf [10.11.2014].

Konigson, Élie, L’Espace théâtral médiéval, Paris, Éditions du CNRS, coll. «Le Chœur des muses », 1975.

LECUPPRE-DESJARDIN, Élodie, La Ville des cérémonies. Essai sur la communication politique dans les anciens Pays-Bas bourguignons, Turnhout, Brepols, coll. «Studies in European urban history », n 4, 2004.

RIVAUD, David, «Les entrées royales dans les "bonnes villes" du Centre-Ouest aux $\mathrm{XV}^{\mathrm{e}}$ et $\mathrm{XVI}^{\mathrm{e}}$ siècles : théâtre et décors historiés »dans La ville au Moyen Âge, vol. 2: Sociétés et pouvoirs dans la ville, dir. Noël COULET et Olivier GuYOTJANNIN, Paris, Éditions du CTHS, coll. «Actes du congrès national des sociétés savantes, section d'histoire médiévale », vol. 120/2, 1998, p. 277-294.

Rousse, Michel, La Scène et les Tréteaux. Le théâtre de la farce au Moyen Âge, Orléans, Paradigme, coll. «Medievalia », n 50, 2004. 


\section{Processions}

Glenisson DelanneE, Françoise, «Fête et société : l'Assomption à Sienne et son évolution au cours du $\mathrm{XVI}^{\mathrm{e}}$ siècle ", dans Les Fêtes urbaines en Italie à l'époque de la Renaissance, dir. Françoise DeCRoisette et Michel Plaisance, Paris, Klincksieck/Presses Sorbonne Nouvelle, coll. «Actes et colloques », n 39, 1994, p. $65-129$.

LECUPPRE-DESJARDIN, Élodie, «La Grande Procession de Lille à la fin du Moyen Âge: entre dévotion populaire et enjeux de pouvoir», dans Sentiments religieux et piété populaire de l'an mil à nos jours, dir. Jean HEUCLIN et Christophe LEDUC, Revue du Nord, hors-série n ${ }^{\mathrm{O}} 25,2011$, p. 43-56.

Moving subjects. Processional performance in the Middle Ages and the Renaissance, dir. Kathleen Ashley, Wim Husken, Amsterdam, Atlanta, Rodopi, coll. «Ludus », $\mathrm{n}^{\mathrm{o}} 5,2001$.

\section{Fête et littérature}

AubAILly, Jean-Claude, "Théâtre médiéval et fêtes calendaires ou l'histoire d'une subversion », Between Folk and Liturgy, dir. Alan J. FlETCHER et W. HÜSKEN, Amsterdam et Atlanta, Rodopi, coll. «Ludus : Medieval and Early Renaissance Theatre and Drama », $n^{0} 3,1997$, p. 31-64.

BADEL, Pierre-Yves, "Le Jeu de la Feuillée est une fête », Études de langue et littérature françaises de l'université d'Hiroshima, 18, 1999, p. 1-15.

Berthelot, Anne, «Des fêtes arthuriennes classiques aux fêtes ritualisées du Roman de Perceforest », Feste und Feiern im Mittelalter. Paderborner Symposion des Mediävistenverbandes, dir. Detlef Altenburg, Jörg JARnUT et Hans-Hugo STEINHOFF, Sigmaringen, Jan Thorbecke Verlag, 1991, p. 432-440.

BOUSMAR, Éric, «La place des hommes et des femmes dans les fêtes de cour bourguignonnes (Philippe le Bon - Charles le Hardi)», dans À la cour de Bourgogne. Le duc, son entourage, son train, dir. Jean-Marie CAUCHIES, Turnhout, Brepols, coll. «Burgundica », $\mathrm{n}^{\circ}$ 1, 1998, p. 11-31.

Bozzolo, Carla et Hélène Loyau, La Cour amoureuse dite de Charles VI, Paris, Le Léopard d'Or, 3 t., t. I, 1982, t. II-III, 1992.

CARON, Marie-Thérèse, « 17 février 1454 : le Banquet du Vœu du Faisan, fête de cour et stratégies de pouvoir », Revue du Nord, vol. 78, n 315, 1996, p. 269-288.

FInOLI, Anna Maria, "Fête de cour et fête de ville dans le Chevalier errant de Thomas III de Saluces (1356? -1416) », Fifteenth Century Studies, n ${ }^{0} 19,1992$, p. 33-53.

FERre, Rose-Marie, "Les ecclésiastiques et les fêtes profanes à la cour de René d'Anjou: l'exemple du Pas de Saumur», L'Artiste et le Clerc. Commandes artistiques des grands ecclésiastiques à la fin du Moyen Âge (XIV ${ }^{e}-X V I^{e}$ siècles), dir. Fabienne JOUBERT, Paris, Presses de l'Université de Paris-Sorbonne, coll. «Cultures et civilisations médiévales », $\mathrm{n}^{\mathrm{o}} 36,2006$, p. 351-370. 
LAFortune-MARTEl, Agathe, Fête noble en Bourgogne au XV siècle. Le banquet du faisan, 1454: aspects politiques, sociaux et culturels, Montréal, Bellarmin, coll. «Cahiers d'études médiévales », nº 8, 1984.

LARDELliER, Philippe, Les Miroirs du paon. Rites et rhétoriques politiques dans la France de l'Ancien Régime, Paris, Champion, coll. «Études et essais sur la Renaissance », $\mathrm{n}^{\mathrm{o}}$ 44, 2003.

Le PERSON, Marc, «Entre critique, dérision et subversion de l'autorité et de la loi : Le jeu de la feuillée d'Adam de la Halle (XIII siècle) est-il une Fête des Fous dramatisée? », Le Moyen Âge et la Renaissance face aux lois : de la critique à la subversion, Cahiers du CELEC, $\mathrm{n}^{\circ} 8,2014$.

MüHLETHALER, Jean-Claude, «Entre prose et vers, exemplaire et éphémère. Regards croisés sur une fête à la cour de Charles VI (Michel Pintoin - Thomas de Saluces Eustache Deschamps), dans L'Euvre littéraire du Moyen Âge aux yeux de l'historien et $d u$ philologue, dir. Ludmilla EvDOLIMOVA et Victoria SMIRNOVA, Paris, Classiques Garnier, coll. «Rencontres », $n^{0}$ 77, 2014, p. 8-97.

Moran, Patrick, «L'épisode de la Forêt Perdue dans le Lancelot en prose : jeux et divertissements périlleux en terre de Bretagne », La Règle du jeu, Questes, ${ }^{\circ}{ }^{0} 18$, p. $87-102$.

PiageT, Arthur, «La cour amoureuse dite de Charles VI », Romania, $\mathrm{n}^{\mathrm{o}} 20,1891$, p. $422-423$.

POIRION, Daniel, Le Poète et le Prince. L'évolution du lyrisme courtois de Guillaume de Machaut à Charles d'Orléans, Paris, Presses Universitaires de France, coll. «Publications de la faculté des Lettres et Sciences Humaines de l'Université de Grenoble », nº 35, 1965.

STANESCO, Michel, «Le banquet du faisan : de la fête courtoise au scénario rituel », Rencontres médiévales en Bourgogne (XIV ${ }^{e}-X V^{e}$ siècles), $\mathrm{n}^{\mathrm{o}}$ 2, 1992, p. 47-67.

UHL, Patrice, «Où faire la fête entre femmes à Paris vers 1320. Une relecture des Trois dames de Paris de Watriquet de Couvin», dans Écritures de la ville, dir. Christian Chelebourg et Serge Meitinger, Paris, Kimé, coll. «Cahiers de recherches sur l'écriture et l'espace », $\mathrm{n}^{\circ}$ 1, 2006, p. 89-107.

WALter, Philippe, La Mémoire du temps, fêtes et calendriers de Chrétien de Troyes à la Mort Artu, Paris/Genève, Champion/Slatkine, coll. "Nouvelle bibliothèque du Moyen Âge », nº 13, 1989.

\section{Fête, violence et ségrégation}

BoItEuX, Martine, «Les Juifs dans le Carnaval de la Rome moderne », Mélanges de l'Ecole française de Rome. Moyen Âge, Temps modernes, vol. 88, n 2, 1976, p. 745-787.

LECUPPRE-DESJARDIN, Élodie, « De l'invective à la prise de conscience identitaire : la guerre entre Douai et Lille (1284-1285)», dans Convaincre et persuader. Communications et propagande aux XII et XIII siècles, dir. Martin AURELL, Poitiers, CESCM, coll. «Civilisations médiévales », nº 18, 2007, p. 415-433. 
WenNinger, Markus J., «Das gefährliche Fest. Ostern als zeitlicher Kristallisationspunkt antijüdischen Verhaltens », dans Feste und Feiern im Mittelalter. Paderborner Symposion des Mediävistenverbandes, dir. Detlef AltenbuRG, Jörg JARNUT et Hans-Hugo STEINHOFF, Sigmaringen, Jan Thorbecke Verlag, 1991, p. 323-332.

\section{La fête dans la ville}

BÄCHTOLD, Jakob, «Das glückhafte Schiff von Zürich : nach den Quellen des Jahres 1576 », Mitteilungen der Antiquarischen Gesellschaft in Zürich, vol. 20, n 2, 1880, p. 84-139.

LECUPPRE-DESJARDIN, Élodie, « De l'invective à la prise de conscience identitaire : la guerre entre Douai et Lille (1284-1285)», dans Convaincre et persuader. Communications et propagande aux XII et XIII siècles, dir. Martin AuRELL, Poitiers, CESCM, coll. «Civilisations médiévales », $n^{\circ}$ 18, 2007, p. 415-433.

-, «La grande Procession de Lille à la fin du Moyen Âge : entre dévotion populaire et enjeux de pouvoir », dans Sentiments religieux et piété populaire de l'an mil à nos jours, dir. Jean HeuClin et Christophe Leduc, Revue $d u$ Nord, hors-série $\mathrm{n}^{\mathrm{o}} 25$, 2011, p. 43-56.

REUSS, Rodolphe, Le Grand Tir strasbourgeois de 1576 et la venue des Zurichois à Strasbourg. Étude historique, Strasbourg, Treuttel et Würtz, 1876.

ThiBAult, Jean, «Fête et renouveau de la vie sociale à Orléans après 1429 », Le Moyen Âge, vol. 116, n 2, 2010, p. 385-406.

VON HEUSINGER, Sabine, «Die Handwerksbruderschaften in Straßburg», dans Städtische Gesellschaft und Kirche im Spätmittelalter, dir. Sigrid SCHMITT, Stuttgart, Steiner, coll. « Geschichtliche Landeskunde », nº 62, 2008, p. 123-140.

\section{En Italie}

Les Fêtes urbaines en Italie à l'époque de la Renaissance, dir. Françoise DECROISETTE et Michel PlaISAnCE, Paris, Klincksieck/Presses Sorbonne Nouvelle, coll. «Actes et colloques », $\mathrm{n}^{\mathrm{o}} 39,1994$.

RIzZI, Alessandra, «Le jeu dans les villes de l'Italie médiévale », Histoire urbaine, 1, 2000-2001, p. 47-64.

\section{Florence}

JASER, Christian, «Agonale Ökonomien. Städtische Sportkulturen des 15. Jahrhunderts am Beispiel der Florentiner Palio-Pferderennen », Historische Zeitschrift, $\mathrm{n}^{\mathrm{o}} 298 / 3,2014$, p. 593-624.

PlaisAnCE, Michel, «Le carnaval des Médicis: de Laurent à François » dans Florence and Milan. Comparisons et relations, dir. Craig Hugh SMYTH et Giancarlo GARFAGnini, Firenze, La Nuova Italia editrice, coll. «Villa i Tatti », n 11,1989, vol. 2, p. 243-255. 


\section{Rome}

Martine BoiteuX, «Les Juifs dans le Carnaval de la Rome moderne », Mélanges de l'Ecole française de Rome. Moyen Âge, Temps modernes, vol. 88, $\mathrm{n}^{\mathrm{o}} 2$, 1976, p. 745-787.

-, «Chasse aux taureaux et jeux romains de la Renaissance », dans Les Jeux à la Renaissance, éd. Philippe Ariès, Paris, Vrin, 1982, p. 33-53.

-, «Lieux de fête et lieux de pouvoir dans l'espace public romain ; le palais, la ville, le peuple » dans Mégapoles méditerranéennes, dir. Claude NICOLET, Robert ILBERT et Jean-Charles DePaule, Paris, Maisonneuve et Larose, Maison méditerranéenne des sciences de l'homme, École française de Rome, 2000, p. 311-350.

\section{Sienne}

GLENISSON DelanNEE, Françoise, «Fête et société : l'Assomption à Sienne et son évolution au cours du $\mathrm{XVI}^{\mathrm{e}}$ siècle », dans Les Fêtes urbaines en Italie à l'époque de la Renaissance, dir. Françoise DeCROISETTE et Michel PlaisAnCE, Paris, Klincksieck/Presses Sorbonne Nouvelle, coll. «Actes et colloques », n 39, 1994, p. 65-129.

Il Mulino delle vanità. Lusso e cerimonie nella Siena medievale, dir. Maria Assunta CEPPARI RIDOLFI et Patrizia TURRINI, Siena, Il Leccio, 1996.

Il Palio di Siena, dir. Giovanni CECCHINI et Dario NeRI, Milano, Electa, 1958.

Il Palio. La festa della città, dir. Luca BeTti et Alessandro FALASSI, Betti, Siena, 2003.

KEMPERS, Bram, «Icons, altarpieces, and civic ritual in Siena cathedral, 1100-1530» dans City and spectacle in Medieval Europe, dir. Barbara HaNAWALT et Kathryn REYERSON, Minneapolis, University of Minnesota Press, coll. «Medieval studies in Minnesota », $n^{0}$ 5, 1994, p. 89-136.

Pertici, Petra, La Città magnificata : interventi edilizi a Siena nel Rinascimento: l'Ufficio dell'ornato, 1428-1480, Siena, Il leccio, coll. « Sena vetus », nº 5, 1995.

\section{Venise}

BerTRAND, Gilles, Histoire du carnaval de Venise : XI $I^{e}-X X I^{e}$ siècles, Paris, Pygmalion, 2013.

BURKE, Peter, «Le carnaval de Venise : esquisse pour une histoire de longue durée » dans Les Jeux à la Renaissance, dir. Philippe ARIES, Paris, Vrin, 1982, p. 55-61.

MuIR, Edward, Civic Ritual in Renaissance Venice, Princeton, Princeton University Press, 1986.

Venise en fêtes, dir. Giandomenico Romanelli, Lina Urban et Fiora GANDOLFI, Paris, Chêne, 1992.

\section{La fête dans le Saint-Empire}


CAuchIES, Jean-Marie, " "Service" du prince, "sûreté" des villes : à propos de privilèges délivrés aux confréries ou serments d'archers et d'arbalétriers dans les Pays-Bas au XV siècle », Revue du nord, 2012, vol. 94, nº 395, 2012, p. 419-434.

Delle LuCHE, Jean-Dominique, «Schützenfeste und Schützengesellschaften in den Residenzstädten: Konfigurationen zwischen Stadt und Fürsten im 15. und 16. Jahrhundert (Pforzheim, Würzburg, Ansbach, Stuttgart)», dans In der Residenzstadt. Funktionen, Medien, Formen bürgerlicher und höfischer Repräsentation, dir. Jan HIRSCHBIEGEL et Werner PARAVICINI, Ostfildern, Thorbecke, 2014, p. 157-174.

Edelmann, August, Schützenwesen und Schützenfeste der deutschen Städte vom 13. bis zum 18. Jahrhundert, München, Pohl, 1890.

Gerhard FouQuET, « Das Festmahl in den oberdeutschen Städten des Spätmittelalters. $\mathrm{Zu}$ Form, Funktion und Bedeutung öffentlichen Konsums », Archiv für Kulturgeschichte, vol. 74 (1992), p. 83-123.

"Kurzweil viel ohn' Maß und Ziel”. Alltag und Festtag auf den Augsburger Monatsbilder der Renaissance, dir. Pia-Maria GRÜBER, München, Hirmer, 1994.

Kuzudo, Kazuhiko, «Open Shooting Festivals (Freischießen) in German Cities, 1455-1501 », The International Journal of the History of Sport, vol. 3, n ${ }^{\circ}$ 1, 1999 p. $65-86$.

MÄND, Anu, Urban Carnival: festive culture in the hanseatic cities of the eastern Baltic, 1350-1550, Turnhout, Brepols, coll. «Medieval texts and cultures of Northern Europe », $\mathrm{n}^{\circ} 8,2005$.

MoNNET, Pierre, «La ville en fête : conceptions et représentations à Francfort (et dans quelques autres villes d'Empire) à la fin $\mathrm{du} \mathrm{XV}^{\mathrm{e}}$ siècle », dans La ville à la Renaissance. Espaces, représentations, pouvoirs $\left(\mathrm{XXXIX}{ }^{\mathrm{e}}\right.$ colloque international d'études humanistes), dir. Gérald CHAIX, Paris, Champion, 2003, p. 11-30.

OSTERMANN, Markus, «"Vmb kurczweil vnd schiessens willen". Zu den gedruckten Schützenbriefen des 15. Jahrhunderts », dans Einblattdrucke des 15. und frühen 16. Jahrhunderts : Probleme, Perspektiven, Fallstudien, dir. Volker HonEMANN, Tübingen, Niemeyer, 2000, p. 397-443.

RADLKOFER, Max, "Die Schützengesellschaften und Schützenfeste Augsburgs im 15. und 16. Jahrhundert », Zeitschrift des Historischen Vereins für Schwaben und Neuburg, $\mathrm{n}^{\mathrm{o}} 21,1894$, p. 87-139. 